\title{
Statistik im Sozialismus
}

\section{Amtliche Daten zwischen Realität und Ideologie und ihre Medienrezeption}

\author{
Walter Krämer · Klaus Leciejewski
}

Angenommen: 21. Mai 2021 / Online publiziert: 18. Juni 2021

(C) Der/die Autor(en) 2021

Zusammenfassung Dieser Beitrag dokumentiert eine Tendenz totalitärer Gesellschaftssysteme, die Statistik und insbesondere Daten der Amtsstatistik als Stütze von Ideologien zu missbrauchen. Dieser Missbrauch wird oft durch westliche Medien unterstützt, die allzu blauäugig auf dergleichen Lügen hereinfallen. Hier versprechen das Internet und die leichte Verfügbarkeit von Massendaten aller Art, ein mögliches Gegengewicht zu werden.

Schlüsselwörter Amtsstatistik $\cdot$ Manipulation $\cdot$ Kuba $\cdot$ DDR $\cdot$ Medien

JEL C80 - C83

\section{Statistic under socialism}

Official data as a mix of reality and ideology and their reception by the media

\begin{abstract}
We establish a tendency in totalitarian regimes to use official statistical data for propaganda purposes. This is facilitated by an equally obvious tendency among western media to take such figures at face value. However, the big data revolution promises easy checks of such false claims and might help impeding such abuses.
\end{abstract}

\footnotetext{
Walter Krämer $(\bowtie)$

Fakultät Statistik, Technische Universität Dortmund, 44221 Dortmund, Deutschland

E-Mail:walterk@statistik.tu-dortmund.de

Klaus Leciejewski

Erftstadt, Deutschland
} 


\section{Einleitung}

Statistik ist ein Vielfacettenwort. Die Rede ist hier von Statistik im ursprünglichen Sinne als der Wissenschaft der quantitativen Staatsangelegenheiten (das lateinische statisticum heißt ,den Staat betreffend“), so wie sie im 18. Jahrhundert an vielen europäischen Universitäten heimisch geworden ist. Daten zu erheben über Menschen, Wirtschaft und Gesellschaft war zwar schon in den alten Ägypter- und Römerreichen üblich, aber daraus eine Wissenschaft zu machen, erachteten in Deutschland erst Gelehrte wie Hermann Conring oder Gottfried Achenwall um 1700 als eine vordringliche Aufgabe ihrer Zeit. Aber diese Daten dann zur Verbreitung einer Ideologie und zur Etablierung eines vermeintlichen Himmelreichs auf Erden zu benutzen, scheint erst mit den totalitären Diktaturen des 20. und 21. Jahrhunderts zu einer immer wieder stattgegebenen Versuchung geworden zu sein

In offenen Gesellschaften haben Daten einen Wert an sich; sie sind Leuchttürme, Leitplanken und Wegweiser, oder sollten es nach allgemeinem Konsens zumindest sein, sie helfen den Bürgern und der Politik, sich in der Welt zurechtzufinden. Als Produkte menschlichen Handelns sind sie zwar kaum jemals frei von Fehlern (siehe dazu auch den folgenden Abschn. 2), und auch an ihrem Verständnis mangelt es, aber trotz aller nicht zu ignorierenden diesbezüglichen Defizite (siehe etwa Bauer et al. 2014) sieht sich die Amtsstatistik eher als Bote und nimmt den Datennutzern die Interpretation nicht ab. In totalitären Gesellschaften dagegen sind Daten und insbesondere die Daten der Amtsstatistik vor allem ein Mittel zum Zweck. Man schätzt sie auf Seiten der Regierenden und schreckt auch vor Fälschungen nicht zurück, um die Schutzbefohlenen auf einen vermeintlich rechten Weg zu lenken. Daten sind Herrschaftswissen, und den Untertanen nur soweit zugänglich zu machen, wie es den Interessen einer vermeintlich besseren Gesellschaft dient.

Wie schon Lenin im Umfeld der russischen Volkszählung 1919 dekretierte, muss Statistik „ein Organ des sozialistischen Aufbaus sein“: „Wir müssen die Statistik in die Massen tragen, sie popularisieren, damit die Werktätigen allmählich selbst verstehen und sehen lernen, wie und wieviel man arbeiten muss“" (siehe Bomsdorf und Krämer 2011). Als dann die 1937er Volkszählung Resultate erbrachte, die die Siegesmeldungen der Partei widerlegten, ließ Stalin die Statistiker erschießen.

Das sollte, wenn man das Wesen totalitärer Herrschaftsformen kennt, auch niemanden überraschen. Überraschend und verstörend ist allein, wie bereitwillig sich freie Gesellschaften oft durch allerlei von totalitären Diktaturen errichtete Potemkinsche Dörfer blenden lassen. Das fing mit den Heerscharen westlicher Intellektueller an, die in den 1930er-Jahren, als Millionen Russen Hungers starben, nach Moskau reisten und an der Grenze, sich an der Tür zum Paradiese wähnend, ihre mitgebrachten Lebensmittel ostentativ liegen ließen (siehe Hollander 1981), und hört mit den modernen, im fünften Kapitel näher beschriebenen Kuba-Verehrern auf.

Der vorliegende Aufsatz will bewirken, dass dies in Zukunft weniger oft geschieht. Der nächste Abschnitt geht kurz auf die allgemeine statistische Adäquationsproblematik ein, die darin besteht, dass es intrinsisch fast unmöglich ist, die Welt und Umwelt mit ihrer Sozialgestalt und Wirtschaftsleistung in eindeutigen, perfekt zu den Begriffen passenden statistischen Zahlen abzubilden. Dieses Problem betrifft offene wie totalitäre Gesellschaften in gleicher Weise, und auch die 
Anreize für die jeweils Regierenden, die hier zahlreich vorhandenen Freiheitsgrade zu ihren Gunsten auszunutzen, ist in beiden Systemen gleich. Allerdings sorgen die Meinungs- und Pressefreiheit hinreichend gut dafür, dass einschlägige Exzesse in freien Gesellschaften unter Kontrolle und überschaubar bleiben.

Dergleichen Exzesse sind in totalitären Systemen die Regel und werden in den folgenden Abschn. 3 und 4 an den Fallbeispielen DDR und Kuba näher aufgezeigt. Wie diese Propaganda dann von gewissen Medien, ja sogar von Forschungsinstituten in Deutschland verbreitet wurde und im Fall von Kuba immer noch verbreitet wird, ist Gegenstand von Abschn. 5. Nur zu bereitwillig werden statistische Falschmeldungen, falls aus ideologisch nahestehenden Quellen kommend, vergleichsweise unkritisch übernommen und als Wahrheiten verkauft. Für die DDR betrifft dies beispielsweise die bekannten Märchen von Millionen neuer Wohnungen und massenweise produzierten Industrierobotern, inklusive der zehntgrößten Wirtschaftsmacht der Welt. Und als immer noch aktuelles Beispiel weisen wir im Fall von Kuba die vielfach im Westen kolportierten Zahlen zu Alphabetisierungsgrad und Säuglingssterblichkeit als Fälschungen bzw. als nicht belegbare Propagandathesen nach. Ein abschließender Abschn. 6 schließlich wirft einen Blick in die Zukunft der staatlichen Datenmanipulation im Kontext des Internets und allgemein verfügbarer Massendaten aller Art.

\section{Das statistische Adäquationsproblem und seine unterschiedliche Bewältigung in offenen und totalitären Gesellschaften}

Es gibt keine von höheren Mächten oder internationalen Gremien verbindlich vorgeschriebene Definition von Phänomenen wie Armut, Reichtum, Kriminalität, Gesundheit, Krankheit oder Arbeitslosigkeit. In allen diesen Fällen versucht die Amtsstatistik mit zum Teil erheblichem Aufwand, sinnvolle und akzeptable Definitionen und Messverfahren zu finden, aber letzten Endes ist nicht zu übersehen, dass alle diese Begriffe auf verschiedene gleichermaßen sinnvolle Arten und Weisen in Zahlen übersetzbar sind. Nimmt man dann noch das umfangreiche statistische Datenmaterial aus der nichtamtlichen Statistik hinzu, die in ihrer Arbeit viel ungebundener und zuweilen sogar ganz offen gewissen Interessen verpflichtet ist, so sollte es niemanden verwundern, dass die so entstehenden Freiheitsgrade von interessierter Seite zumindest versuchsweise gerne ausgelotet werden. In Krämer (2003) etwa wird gezeigt, wie das in der bundesdeutschen Definition von Arbeitslosigkeit verwendete Kriterium „dem Arbeitsmarkt unmittelbar zur Verfügung stehen“ von den jeweils Regierenden dazu verwendet wird, die amtlich gemessenen Arbeitslosenzahlen klein zu rechnen. Nicht unmittelbar zur Verfügung stehen etwa Arbeitnehmer, die krankgeschrieben sind (soll in den Niederlanden eine bewährte Methode zur Reduzierung der Arbeitslosenzahlen sein, siehe Koning und Van Vuuren (2006)), Teilnehmer von Umschulungs- oder Weiterbildungsmaßnahmen oder Strafgefangene. Auf diese Weise reduzieren etwa die USA ihre potenzielle Arbeitslosenzahl um über zwei Millionen Personen.

In Deutschland waren lange Zeit vor allem Umschulungs- und Fortbildungsmaßnahmen sowie Erziehungszeiten ein Stein des Anstoßes für alle, die gerne höhere 
Arbeitslosenzahlen gesehen hätten (also die jeweilige Opposition); durch die Verlängerung des Erziehungsurlaubs von 6 auf 10 Monate ließ etwa die Regierung Kohl in den 1980ern auf einen Schlag 100.000 arbeitslose Frauen aus der Statistik verschwinden. Ein Dutzend Jahre später machte die jetzt regierende Opposition das gleiche und weitete die Möglichkeiten für Umschulungen und Frühpensionierungen beträchtlich aus.

Fast schon grotesk ist auch der öffentliche und mediale Umgang mit der Messung der Armut hierzulande (Krämer 2000). Die nimmt, wenn man den Medien glauben darf, von Jahr zu Jahr in Deutschland zu. „Jeder achte Deutsche ist arm“ meldete die Welt im Jahr 2008. Acht Jahre später war es schon fast jeder sechste (Die Welt 2014). In Wahrheit geht die Armut in Deutschland, wenn man etwa die Kriterien von A.K. Sen (2011) von Armut als Mangel an Verwirklichungschancen zugrunde legt, von Jahr zu Jahr zurück (siehe etwa Giesselmann und Luekemann (2017) und viele andere Quellen mehr). Eher skurril dagegen mutet die auf der amtlichen Kriminalitätsstatistik beruhende Rangfolge von kriminellen Städten oder Ländern an. So scheinen sich etwa die deutschsprachigen Medien immer mal wieder einen Spaß daraus zu machen, den Vatikanstaat als den nach offizieller Lesart kriminellsten Staat der Erde zu vermelden (siehe etwa den Wiener Standard (2006) und viele andere): Nirgendwo sonst ist der Quotient „Zahl der im Land gemeldeten Straftaten geteilt durch Zahl der Einwohner des Landes“ derart hoch. Und auch der Kriminalitätsvergleich der deutschen Städte leidet unter dieser Normierung durch die Zahl der Einwohner, die besser durch die Zahl der potenziellen Opfer- und Straftätertage zu ersetzen wäre (also Einwohner plus Einpendler plus zeitgewichtete Ein- und Aussteiger an Flughäfen und Bahnhöfen; allein in Frankfurt am Main, der nach dieser Statistik regelmäßig kriminellsten Stadt der Bundesrepublik, kämen so jährlich über 50 Mio. Fluggäste dazu). Aber wie ja schon die Medien durch ihre regelmäßigen belustigten Kommentare zeigen, bleiben solche Adäquationsfehler in offenen Gesellschaften nicht unentdeckt und werden, so darf man hoffen, auf lange Sicht verschwinden.

Anders in totalitären Systemen, wie in den beiden folgenden Abschnitten an den Beispielen DDR und Kuba aufgezeigt. Einmal wird hier das Adäquationsproblem nicht offen diskutiert. So konnte etwa die DDR mehrere Jahre lang immer wieder neue Rekorde bei fertiggestellten Wohnungen oder Industrierobotern melden, aber nur deshalb, weil beide anders definiert waren als im Rest der Welt. Und da, anders als in freien Gesellschaften, über Statistik in totalitären Systemen nicht offen geredet werden darf, konnte man so jahrelang als erfolgreiche Industrienation oder kann man immer noch, wie im Fall von Kuba, als medizinsoziologisches Erfolgsmodell die Welt zum Narren halten. In beiden Systemen waren bzw. sind immer noch internationale Vereinbarungen betreffend Datenqualität und -definition entweder überhaupt nicht oder nur um den Schein zu wahren anerkannt, und das Offenlegen des Zustandekommens amtlicher Statistiken gilt fast schon als eine Zumutung westlicher Industriespione, der man mit Macht entgegentreten muss.

Natürlich kommen auch in vergleichsweise offenen westlichen Statistiksystemen Datenschwindeleien vor. Das bekannteste Beispiel sind die Manipulationen der Schulden- und Außenhandelsstatistiken durch das Generalsekretariat für statistische Dienste Griechenlands, mit denen man sich vor der Jahrtausendwende den Beitritt in 
die Eurozone erschlichen hat (siehe Krämer 2013, Kap. 4). Bekanntlich wurde der Präsident der vermeintlich unabhängigen Nachfolgeorganisation ELSTAT, Andreas Georgiou, wegen der Aufdeckung ähnlicher Verirrungen sogar zu zwei Jahren Haft verurteilt. Aber anders als in der Volksrepublik China, in Kuba, in der DDR, in Russland oder in Nordkorea kommen solche Affären meist ans Tageslicht und zur Kenntnis der Öffentlichkeit.

\section{Fallstudie DDR}

Die amtliche Statistik in der DDR hatte vor allem dem Regime zu dienen, getreu der Maxime des Direktors des Zentralinstituts für sozialistische Wirtschaftsführung Helmut Koziolek (Koziolek et al. 1970. S. 37): „Kein Gebiet des gesellschaftlichen Lebens und kein Tätigkeitsbereich der Menschen kann im Sozialismus ohne politische Einstellung, ohne Bestimmung des politischen Inhalts oder ohne politische Maßnahmen wissenschaftlich geleitet werden. Die Politik der Partei durchdringt alle Sphären des Lebens und die Entwicklung unserer Gesellschaft.“ Oder wie es der seinerzeitige Leiter des Staatlichen Statistischen Zentralamtes Heinz Rauch einmal formulierte (Rauch 1959, S. 169): „Es gibt keine Statistik schlechthin, sondern nur eine bürgerliche und eine sozialistische Statistik.“ Es komme dabei darauf an, „daß wir allen unseren Mitarbeitern [der SZS] die führende Rolle der Partei der Arbeiterklasse klarmachen“, und die Aufgabe der sozialistischen Statistik sei es, „den Werktätigen (zu) helfen, die Überlegenheit unserer sozialistischen Gesellschaftsordnung auf allen Gebieten des Lebens gegenüber der kapitalistischen Ordnung zu erkennen“".

Damit soll nicht bezweifelt werden, dass viele Kollegen der DDR-Amtsstatistik dennoch nach Kräften bemüht waren, die üblichen Standards einer seriösen Datenerhebung einzuhalten. Eine nützliche Übersicht über die Alltagssorgen und -nöte dieser Personen ,,an der Front“ findet sich in Kockel (1992). Aber was nutzt alle Seriosität am Anfang einer statistischen Wertschöpfungskette, wenn dann das Endprodukt einer solchen Datenbeschaffung, wie etwa die DDR-Außenhandelsstatistik, auf Anordnung des Politbüros der SED per Federstrich nach Gusto um einige 100 Mio. Valutamark nach unten oder oben abgeändert wird. Siehe ein einschlägiges Dokument auf S. 669 der Dokumentation von von der Lippe (1996).

Kaschiert wurden solche Manipulationen etwa durch die Praxis, im statistischen Jahrbuch der DDR die Größen der Entstehungs- und Verwendungsseite des Nationaleinkommens nur durch spaltenweise und zeilenweise Prozentanteile ohne Bezugsgrößen oder durch absolute Werte in Valutamark ohne Umrechnungskurs zu veröffentlichen. Damit war es für Interessierte schlechterdings unmöglich, konsistente Ergebnisse abzuleiten ${ }^{1}$.

Auch die Kriminalität in der DDR hatte per Order des Zentralkomitees niedriger zu sein als im Westen, und so wurde sie es denn auch. Einmal durch glatte Fälschungen, oder indem man missliebige Zahlen einfach unterdrückte. Als es etwa $1971 \mathrm{zu}$ einem deutlichen Anstieg der gemeldeten Straftaten kam, wurde deren Veröffent-

\footnotetext{
1 Wir danken einem anonymen Gutachter für diesen Hinweis, den wir hier wörtlich wiedergeben.
} 
lichung auf Anweisung des DDR-Generalstaatsanwaltes bis 1977 ausgesetzt. Dann durch Anpassung der Definition (Mertens 1998, Sensch 2007). So wurden etwa seit dem Inkrafttreten des neuen DDR-Strafgesetzbuches am 1. Juli 1968 bestimmte, im Westen als Straftaten erfasste Delikte wie Bagatelldiebstähle, Beleidigung oder Hausfriedensbruch nicht mehr als Straftaten gezählt.

Besonders weit von der Wahrheit entfernt waren jedoch die Statistiken zu Industrierobotern und fertiggestellten Wohnungen. Auf ihrem VIII. Parteitag 1971 hatte die SED beschlossen, bis 1990 drei Millionen neue Wohnungen zu bauen. Und so wurde dann auch am 12. Oktober 1988 von Erich Honecker in der Erich-Correns-Straße (heute Vincent-van-Gogh-Straße) in Berlin-Neu-Hohenschönhausen die dreimillionste seit 1970 in der DDR gebaute Wohnung feierlich übergeben.

In Wahrheit waren es über eine Million Wohnungen weniger. „In den Veröffentlichungen der ehemaligen DDR zum Wohnungsbau waren bis 1989 in die Anzahl der fertiggestellten Wohnungen auch bereits vorhandene Wohnungen einbezogen, die durch die Erstausstattung mit Bad bzw. Dusche, Innen-WC oder durch Ausstattung mit einer anderen Heizungsart als der Ofenheizung modernisiert wurden. Außerdem umfassten die Angaben zum Wohnungsneubau auch fertiggestellte Gemeinschaftsunterkünfte in Arbeiterwohnheimen und Plätze in Feierabend- und Pflegeheimen sowie Rekonstruktions-, Um- und Ausbauwohnungen. Daraus ergab sich in den DDR-Veröffentlichungen für den Zeitraum 1971 bis 1989 ein überhöhter Ausweis von insgesamt 1,2 Mio. fertiggestellter Wohnungen“" (Statistisches Bundesamt 1993, S. 6).

Ein weiteres ehrgeiziges und dann durch Manipulation der Statistik auch erreichtes Ziel der DDR-Führung war es, als weltweit führende Technologienation zu gelten. Dieser Status war in den 1980er-Jahren eng mit der Anzahl von funktionsfähigen Industrierobotern verknüpft. „Nicht 9000 Industrieroboter, wie ursprünglich auf einer Tagung des Zentralkomitees der SED Ende 1980 festgelegt, und auch nicht 40.000 bis 45.000 Roboter wie noch im April 1981 auf dem X. Parteitag der SED verkündet, sollen bis zum Ende des jetzt laufenden Fünfjahrplanes, bis Ende 1985, produziert und eingesetzt werden. Das Anfang Dezember 1981 veröffentlichte Gesetz über den Fünfjahrplan 1981 bis 1985 sieht verbindlich vor, dass nunmehr insgesamt 45.000 Industrieroboter in der DDR zum Einsatz kommen sollen“ (Computerwoche 1982).

Und die kamen dann auch zum Einsatz und stellten die einschlägigen Zahlen aus dem Westen deutlich in den Schatten - die DDR als führende Technologienation. Aber auch hier war der Erfolg nur Schein und ein Produkt einer nie offengelegten Definition: „Zu den Robotern zählen nach der in der DDR inzwischen wohl gültigen Definition des Begriffes ,Industrieroboter', die Gesamtheit von Grundmitteln, die der selbständigen Handhabung von Werkstücken, Werkzeugen und Materialien zur Automatisierung von Haupt- und Hilfsprozessen dienen'. Eine in dieser Weise gefasste Definition macht es möglich, als Roboter nunmehr auch einfache Handhabungsgeräte zu bezeichnen“ (Computerwoche 1982). 


\section{Fallstudie Kuba}

Anders als die DDR gilt Kuba vielen deutschen Intellektuellen immer noch, wenn auch der Glanz zusehends verblasst, als Vorbild einer menschenwürdigen Gesellschaft. Wie im folgenden Abschn. 5 gezeigt, wirken auch die deutschen Leitmedien bei der Perpetuierung dieses Trugbilds mit. Zwar sieht man sich gezwungen, auch einige unübersehbare Schwachstellen des Systems zu benennen, aber diese eher zaghaft und sozusagen pflichtgemäß vorgetragene Kritik verblasst vor der Bewunderung und Sympathie für eine vermeintlich große soziale Leistung. Weiter unten wird dies an einer Reihe von Beispielen gezeigt.

Die wichtigste Basis dieses Trugbilds ist auch hier ein systematischer Missbrauch der amtlichen Statistik. Wie in allen sozialistischen Staaten hat diese auch in Kuba zwei nicht immer kongruente Aufgaben - Steuerung und Propaganda - zu erfüllen. Die Regierungs- und Verwaltungsspitze will die Wahrheit wissen (oder sollte sie zumindest wissen wollen) und ist deshalb an korrekten Zahlen interessiert. Wie schon bei der Einschätzung der DDR-Statistik wird deshalb oft die Meinung vertreten, dass die Zahlen der sozialistischen Amtsstatistik durchaus glaubwürdig seien, da sie ja auch den eigenen Lenkungsaufgaben dienten. Aber dabei wird sowohl die menschliche Kapazität zum Selbstbetrug als auch die Fähigkeit der sozialistischen Datenverbreiter unterschätzt, zwischen Außen- und Innenbild zu unterscheiden.

Das Außenbild, also in gewisser Weise der Propagandateil der kubanischen Amtsstatistik, besteht aus den offiziellen, von den Vereinten Nationen und bis vor kurzem auch vom Statistischen Bundesamt ungeprüft übernommenen Daten des statistischen Amtes ONEI (Oficina Nacional Estadística e Información). In einem Briefwechsel mit einem der Autoren bestätigte ein Mitarbeiter des Statistischen Bundesamtes, dass auch aus dessen Sicht diese Zahlen nicht valide seien und zumindest mit einem entsprechenden Hinweis versehen werden müssten (O'Donnell 2018²). Inzwischen hat denn auch das Bundesamt seine Kuba-Statistik umgestellt; es gibt nicht mehr die staatlichen kubanischen Zahlen, sondern nur noch die der internationalen Organisationen weiter. Sind diese nicht vorhanden oder ganz offensichtlich zweifelhaft, sind Leerstellen angeführt. Aber es bleibt immer noch die Frage offen: Woher stammen die Daten der internationalen Organisationen? Auch hier sind bei einigen Datenlieferanten wie der International Labour Organization ILO auf Grund langjähriger Nähe zum sozialistischen Kuba Zweifel angebracht. In einem weiteren Briefwechsel mit einem der Autoren (O'Donnell 20203) lehnt das Statistische Bundesamt sozusagen die Verantwortung für die Validität solcher internationalen Daten ab und verweist die Nutzer auf die Datenlieferanten.

Gelingt es, in persönlichen Kontakten Vertrauen zu Kubanern aufzubauen, die über interne Kenntnisse der Entstehung kubanischer Statistiken verfügen, dann geben diese offen zu, dass fast jede kubanische Statistik gefälscht ist. Und kaum jemand in der kubanischen Bevölkerung glaubt daran, niemand nimmt sie ernst. Wer könnte dies denn auch, nach sechs Jahrzehnten unentwegter Jubelmeldungen, nach bis in die Gegenwart hinein großartigen Zahlen über Ernteergebnisse und zugleich leeren

\footnotetext{
2 O'Donnell D (2018): Persönliche Mitteilung

3 O’Donnell D (2020): Persönliche Mitteilung
} 
Märkten. Der Gegensatz der staatlichen Meldungen und der alltäglichen Erfahrungen ist einfach zu groß. Als Propaganda sind die kubanischen Statistiken heute in Kuba wirkungslos. Die Kubaner wissen um die Lüge, aber viele westlichen Analysten glauben sie.

Der Lateinamerikaforscher Carmelo Mesa Lago liefert in etlichen Schriften Belege für diese Fälschungen wie auch für Inkongruenzen innerhalb des offiziellen Zahlenwerks; siehe etwa Mesa Lago (2009) und Pérez-López und Mesa-Lago (2009). So weist er zum Beispiel darauf hin, dass $43 \%$ der Exporte nicht spezifiziert werden, oder dass es keine Zahlen zur Zahlungsbilanz, zur Verschuldung oder zur Akkumulation der ausländischen Investitionen gibt. Immerhin ist die ONEI-Außenhandelsstatistik die einzige mit einer „langen Reihe“, von 1950 bis 2018, wenn auch anfangs nur in 5-Jahres-Intervallen. Für Kuba ist ihre Publikation erstaunlich, denn diese Reihe weist nach, dass das republikanische Kuba regelmäßig einen AuBenhandelsüberschuss erzielte, das sozialistische Kuba hingegen nie. Der für den Fünfjahreszeitraum von 1955 bis 1960 erzielte geringfügige Überschuss ist nicht relevant, weil nur die Jahre 1959 und 1960 bereits „Castro-Jahre“ waren. In den Daten für die Exporte wie für die Importe tauchen zusätzlich die Begriffe „Donaciones“ (direkt übersetzt "Geschenke“) auf, deren Zahlen in den Exporten bzw. Importen enthalten sind. In der Legende wird dieser Begriff nicht erklärt. Dahinter könnten sich "geschenkte" Warenlieferungen verbergen, aber solange die kubanische Regierung dafür keine Spezifikation liefert, bleibt dies Spekulation.

In der Statistik von 2018 sind die Warenexporte in fünf Untergruppen aufgeteilt: Agrarprodukte, Fisch, Zucker, Mineralien, Zigarren, im Gesamtwert von 1292 Mrd. Peso. Der anderswo gemeldete Gesamtexport hat aber einen Wert von $2373 \mathrm{Mrd}$. Peso. Bei den fehlenden rund 1. Mrd. Peso könnte es sich um die schon von Mesa Lago angeführten $43 \%$ handeln. In einer anderen Statistik erfolgt dann eine alternative Aufteilung, die internationalen Standards entsprechen soll: „Exports of goods according to sections of the Standard International Trade Classification (SITC)“. Aber vom Gesamtexport von 2373 Mrd.Peso sind auch darin nur 2169 Mrd. Peso enthalten. Erneut die Frage nach dem Verbleib von 204 Mio. Peso.

Eine Überraschung folgt bei dem Export von Dienstleistungen: „Valor del comercio exterior de servicios exportados por divisiones“. Dieser soll 11.290 Mrd. Pesos betragen, davon mit 6399 allein der ,,salud humana“, was dem lange als ,,Solidarität" verbrämten Export von medizinischem Personal entsprechen könnte. Der Konjunktiv ist hier angebracht, denn auch diese Position wird in der Legende nicht erklärt. Werden nun diese Angaben mit denen der komprimierten Außenhandelsstatistik verglichen, so liegt der angegebene Betrag in der Dienstleistungsstatistik um ein Fünffaches über dem des Warenexports, und müsste damit sowohl das Handelsdefizit von ca. 9 Mrd. Peso ausgleichen als auch zur Begleichung der kubanischen Verbindlichkeiten beitragen. Dies ist jedoch nicht der Fall, denn bereits 2018 konnte Kuba seine Verbindlichkeiten nur teilweise bedienen, und seit Ende 2019 stellte das Land deren Bedienung vollständig ein. Zudem ist es fraglich, ob die in der Dienstleistungsstatistik angeführten Positionen auch tatsächlich realen Einnahmen entsprechen. Als Fazit darf gelten, dass sich die kubanische Statistik in zahlreichen Bereichen selber widerspricht. 
Diese Fälschungen der kubanischen Statistik haben drei aus der DDR bekannte Ursachen. Einmal das statistische Meldewesen, insbesondere bei den Wirtschaftsdaten. Zunächst liefert hier jeder kubanische Betriebsdirektor Zahlen an seine übergeordnete Wirtschaftseinheit ab, die oft nicht den realen Ergebnissen entsprechen er oder sie hat Vorgaben erhalten, von deren Einhaltung die persönliche Stellung abhängt. Genauso gehen die Vorgesetzten vor, gleichfalls die Minister, bis die Zahlen bei der zuständigen Abteilung des Zentralkomitees der Kommunistischen Partei ankommen. Hier wird dann, wie in der DDR, ein Abgleich mit den Wünschen der obersten Führung vorgenommen. Und diese letzte „Justierung“ geht dann zur Freigabe an das statistische Zentralamt weiter.

Die zweite Fälschung ist keine bewusste. Sie ergibt sich aus dem System der kubanischen Doppelwährung. Zwar erfolgen die offiziellen Angaben in Peso (Währungscode CUP), aber die Preise in den größeren Geschäften, in den Hotels oder in den Mietwagenstationen sind in der Regel in CUC angegeben (binnenkonvertible Währung, eins zu eins zum Dollar). In der Bank kostet ein CUC zum Zeitpunkt der Abfassung dieser Zeilen aber 25 CUP, und in Kiosken oder Cafeterien ist diese Relationen oft nochmals ungünstiger für den CUP. Diese Doppelwährung steht einer konsistenten Umrechnung von nominalen in reale Größen entgegen und erschwert damit zutreffende Angaben über Produktionsumfänge. Auch bei Importen und Exporten werden die gemeldeten Nominalgrößen den tatsächlichen Mengenrelationen nicht gerecht. Zwar erfolgte zu Beginn des Jahres 2021 eine lange angekündigte „Währungs-, Lohn- und Preisreform“, aber die Doppelwährung soll noch bis Mitte des Jahres gelten. Unabhängig davon ist inzwischen in weiten Bereichen des Konsums und der Dienstleistungen der US-Dollar zur Erstwährung geworden, da der Staat den Großteil aller Konsumgüter nur noch - über Bankkarten - gegen Dollar verkauft, zu dem angeführten staatlich festgesetzten Kurs von einem Dollar zu 24 kubanischen Peso. Aber bis auf naive Ausländer tauscht in Kuba niemand seine Dollar bei einer staatlichen Bank zu diesem Kurs. Zum Zeitpunkt der Abfassung dieses Artikels liegt der Schwarzmarktkurs bei ca. 1 zu 48, was einer - binnenwirtschaftlichen - Abwertung in wenigen Wochen von $100 \%$ entspricht. Das hat zur Folge, dass die Finanzstrukturen der Unternehmen und die zentralen staatlichen Statistiken nach wie vor die tatsächlichen Aufwendungen und Distributionsverhältnisse nicht richtig wiedergeben.

Die dritte Fälschung ist eine indirekte. Viele Statistiken sind Gefangene ihrer Definition, siehe Abschn. 2. Für die meisten gibt es internationale Vereinbarungen. Kuba ist keiner dieser Vereinbarungen beigetreten. In der kubanischen Statistik bleiben zahlreiche Begriffe ohne Definition, auch nicht in den beigefügten Legenden. So werden etwa die landwirtschaftlichen Unternehmen zwischen Staat und drei ,nichtstaatlichen“ Formen aufgegliedert, aber dann folgt eine Zahl ,andere“. Diese betrifft $12 \%$ aller Unternehmen und bleibt unerklärt. Auch bei fast allen anderen Statistiken finden sich Begriffe, die in den Legenden nicht erklärt werden. Bei dem ,kultivierten“ Land gibt es etwa eine Zahl „Brache“, ohne Erklärung. Das ist verständlich, denn eine Zahl zum Bewuchs von „Marabú“ (tropisches Unterholz) fehlt ebenso; sie ist politisch brisant und deshalb unerwünscht.

Auch auf dem Wohnungsmarkt ist, ähnlich wie in der DDR, den Statistiken nicht zu trauen. Amtlich gemeldet werden fertigstellte „Häuser“, ohne Informationen, ob 
neu oder renoviert, wie viele Wohnungen usw. Wir demonstrieren diese dritte, dem Adäquationsproblem geschuldete Fehlerquelle etwas detaillierter anhand des Alphabetisierungsgrades und der Säuglingssterblichkeit. In der DDR-Statistik etwa lag die Säuglingssterblichkeit stets unter der der Bundesrepublik, bis nach der Wende bekannt wurde, dass diese Zahl ganz anders als im Westen ermittelt wird. So muss etwa ein Säugling, damit er „legal“ sterben kann, zunächst einmal lebend geboren sein. Aber wann ist ein Säugling lebend geboren? Diese Unterscheidung von Lebendund Totgeborenen wurde und wird in Deutschland West durch die „Verordnung zur Ausführung des Personenstandsgesetzes“ (PStV) geregelt. Danach sind „Lebendgeborene" Kinder, bei denen entweder das Herz geschlagen oder die Nabelschnur pulsiert oder die natürliche Lungenatmung eingesetzt hat. Damit orientiert man sich an der Definition der Weltgesundheitsorganisation (WHO 1993): „A live birth is defined to be the complete expulsion or extraction from the mother of a baby, irrespective of the duration of the pregnancy, which, after such separation, breathes or shows any other evidence of life, such as beating of the heart, pulsation of the umbilical cord, or definite movement of the voluntary muscles, whether or not the umbilical cord has been cut or the placenta is attached".

Das war zu anderen Zeiten und ist immer noch an anderen Orten anders. Bis 1957 kam es in Deutschland West nur auf die Lungenatmung an. In der DDR dagegen mussten alle obigen Kriterien gelten, damit ein Kind als lebend geboren zählte. Ein Säugling, dessen Lunge funktionierte, das Herz aber nicht, war in Frankfurt/ Main lebend geboren, und konnte dann auch sterben. In Frankfurt/Oder aber nicht. Unter anderem mit dergleichen Definitionstricks erreicht Kuba eine der weltweit niedrigsten Raten für Säuglingssterblichkeit: aktuell 4,0 Promille, das Minimum in Nord-, Süd- und Mittelamerika; die USA melden 5,9 Promille; siehe United Nations Statistics Division (2020). Aber wie wird diese Zahl gemessen, und wer hat diese Messung überprüft? Die United Nations Statistics Division meldet dazu nur lapidar: „Most of the vital statistics data presented in Tab. 3 come from national civil registration systems. These systems differ markedly in the completeness of the data that they produce“ (2020, S. 2). Und wer bestimmt die Risikopopulation? „Es ist eine Vorgabe des Gesundheitsministeriums an die Gynäkologen und Geburtshelfer, dass auf Kuba weniger werdende Mütter - oder Neugeborene im ersten Jahr - sterben dürfen als sonst irgendwo auf der Welt. Dieses Ziel ist keineswegs unerreichbar, wenn die Mediziner in den pränatalen Untersuchungen extrem strenge Maßstäbe anlegen und beim geringsten Zweifel an der Gebärfähigkeit der Mutter oder an der Gesundheit ihrer Leibesfrucht eine Abtreibung anordnen - nicht etwa nur empfehlen. Gegen eine solche Anweisung hat die kubanische Patientin kein Einspruchsrecht" (Widmann 2012, S. 146).

Auch die Definition des zentralen Kriteriums „lebend geboren“ ist nicht klar. Wie etwa Woods et al. (2014) zeigen, differieren hier die Maßstäbe selbst zwischen Krankenhäusern ein- und desselben amerikanischen Bundesstaates, allen Vorgaben der WHO zum Trotz. Und nochmals größer ist die Variation zwischen nationalen statistischen Systemen. Alesina und Redmont (2005) etwa dokumentieren große artifizielle Unterschiede, mit in der Regel groben Unterschätzungen der Säuglingssterblichkeit, in den Nachfolgestaaten der ehemaligen Sowjetunion, und Gourbin und Masuy-Strooband (1994) finden ähnliche Differenzen auch innerhalb der EU. 
Besonders grob werden Falschmeldungen in Sinn von Unterschätzungen auch in gewissen Ländern Mittel- und Südamerikas; wie die Vereinen Nationen recht verschämt in einer Fußnote ihres Demographic Yearbook anmerken (United Nations 2019, S. 109), sind dort die Eintragungen in das Melderegister oft noch mit dem Akt der Taufe kombiniert; Säuglinge, die vorher sterben, waren niemals da und gehen damit auch nicht in die Statistik der Säuglingssterbefälle ein.

Es versteht sich von selbst, dass diese Statistik damit für internationale Vergleiche ungeeignet ist. Damit soll nicht angezweifelt werden, dass Kuba zu den Staaten mit einer niedrigen Kinder- und Säuglingssterblichkeit gehört. Aber es plakatiert diese Ziffer als einen Vorteil seines sozialistischen Systems und lässt keine Überprüfung $\mathrm{zu}$.

Ähnlich ist es mit seiner Alphabetenrate, die bei den über 15-jährigen bei 99,8\% liegen soll (CIA The World Factbook). Kuba gehört damit zu den zehn Ländern mit der weltweit höchsten Rate an Menschen, die lesen und schreiben können. Die anderen neun sind Andorra, Australien, Grönland, Israel, Kirgisistan, Nordkorea (da beträgt die Alphabetisierungsrate laut CIA sogar 100\%), Kasachstan, Tadschikistan und Usbekistan.

Ohne Hintergrundwissen sind solche Zahlen ohne Aussagekraft. Das weiß auch die CIA, die ihre Zahlen ungeprüft von der UNO übernimmt: „Detailing the standards that individual countries use to assess the ability to read and write is beyond the scope of the Factbook" (CIA 2020). So gibt es in jedem Land eine bestimmte Anzahl Menschen, die zwar in gewisser Weise bildungs-, aber nicht lernfähig sind. In Deutschland sollen rund zwei Millionen Erwachsene weder lesen noch schreiben können. Andere gehen von 7,5 Mio. „funktionaler Analphabeten“ aus (Grotlüschen und Rieckmann 2011). Dabei kommt es natürlich auf die Kriterien wie auch auf die Art und Weise ihrer Erfassung an. In Deutschland lässt sich dies ermitteln, wenngleich zuweilen nur durch mühsames Recherchieren, in Kuba nicht. Hier gibt es zwar flächendeckend Schulen, häufig als Internat, in denen lernunfähige Kinder betreut werden und ein begrenztes Wissen vermittelt erhalten. Wenn ein Kind nach Abschluss dieser Schule seinen Namen schreiben kann, aber nicht viel mehr, ist es in der kubanischen Statistik ein Alphabet. So kann kein entwickelter Industriestaat mit einer adäquaten Definition diese kubanische Ziffer von 99,8\% Alphabeten jemals auf seriöse Art erreichen (und die Zahl von $100 \%$ in Nordkorea erst recht nicht).

Auch eine andere Überschlagsrechnung entlarvt die kubanische Alphabetenrate als Illusion. Laut UNESCO (2020) besuchen $92 \%$ aller kubanischen Kinder eine Grundschule, von denen wiederum $96 \%$ den Schulabschluss erreichen. Träfen diese Zahlen zu, wäre die kubanische Erfolgsmeldung von 99,8\% Alphabeten auch dadurch widerlegt. Es wird jedoch noch verwirrender: In der Volkszählung von 2012 gaben acht Prozent aller Kubaner an, über keine Schulbildung zu verfügen. Auch das verträgt sich nicht mit 99,8\% Alphabeten in der Bevölkerung. Wird von einer Alphabetenrate von $76 \%$ vor der Revolution ausgegangen (Marrero 1957), dann ist der inzwischen erreichte Stand von vermutlich knapp über 85\% nach 60 Jahren kein besonderer Erfolg. Eine Überschlagsrechnung der Ergebnisse der letzten Volksrechnung und der UNESCO-Zahlen sowie der Zahlen der österreichischen Außenhandelskammer findet sich auch in Leciejewski (2020). Die Revolution hät- 
te zwar eine Steigerung erreicht, die aber in anderen lateinamerikanischen Staaten ohne eine sozialistische Revolution ebenfalls erreicht wurde.

\section{Die Rolle der Medien und ausgewählter Stiftungen und Forschungsinstitute}

Das statistische Trugbild, mit dem die DDR jahrzehntelang den Rest der Welt zum Narren hielt, lebte auch von der Hilfe gutgläubiger Unterstützer im Westen. Diese Zusammenhänge sind etwa in Hacker (1992), von der Lippe (1995, 1996), Buck (1998) oder Roesler (2019) ausführlich beschrieben. Eine wichtige Rolle spielte dabei das Deutsche Institut für Wirtschaftsforschung (DIW) in Berlin, wo eine Referatsleiterin quasi im Alleingang das bundesrepublikanische Wirtschaftsbild der DDR bestimmte. Einer der Autoren dieser Studie hat sie nach seiner Ausweisung aus der DDR Anfang 1987 im DIW besucht und angeboten, als habilitierter Wirtschaftshistoriker bei der Aufklärung der wahren Datenlage zu helfen, musste aber erfahren, dass daran kein Interesse bestand. Dennoch wurde unter ihrer Leitung das vom DIW Berlin herausgegebene DDR-Handbuch in vielen Auflagen - auch einer englischen - fortentwickelt. „Es war eine weit verbreitete Informationsquelle, insbesondere in der politischen Bildung“ (DIW 2009).

„Desinformationsquelle“ hätte die Wahrheit besser getroffen. Von der Lippe (1995) weist anhand zahlreicher Originaldokumente nach, wie sich das DIW als Sprachrohr der SED missbrauchen ließ. Auch Roesler (2019) stellt in seiner Analyse der Wendemonate dem DIW kein gutes Zeugnis aus: „Legt man die aus den internen Unterlagen gewonnen Erkenntnisse über den Zustand der DDR-Wirtschaft und ihrer Wirtschaftspolitik in den ersten drei Quartalen des Jahres 1989 zugrunde, so kann man sich des Eindrucks nicht erwehren, dass die eingangs zitierten DDRHistoriker die Wirtschaftslage und die Aktivitäten der DDR-Führung zur Steuerung der Wirtschaft zu negativ darstellen. Gleichzeitig wird deutlich, dass das westdeutsche Wirtschaftsinstitut DIW sie seinerzeit jedoch zu positiv einschätzte." In Cornelsen (1989) ist etwa zu lesen: „Die DDR-Wirtschaft hält längerfristig ein Wirtschaftswachstum von 4 v.H. jährlich für erforderlich. Diese Rate wurde auch dem Volkswirtschaftsplan für das Jahr 1989 zugrunde gelegt. Nach dem Bericht über das erste Halbjahr 1989 ist bisher das vorgesehene Wachstumstempo erreicht worden“.

Vielleicht auf dem Papier. Selbst kurz vor dem Zusammenbruch wird im DIW noch Planerfüllung konstatiert. Und auch in zahlreichen anderen Publikationen (siehe etwa Cornelsen 1984 oder Cornelsen et al. 1984) stellt sich das DIW in Hinsicht auf objektive Analyse und Berichterstattung kein gutes Zeugnis aus.

Im Fall von Kuba dauert diese Desinformation noch heute an. Eher unfreiwillige Helfer sind hier die statistischen Ämter vieler Staaten und der Vereinten Nationen, ja sogar der amerikanische Geheimdienst CIA: „In der Tat hat das Land die niedrigste Säuglingssterblichkeit und die höchste Lebenserwartung unter den großen Staaten Lateinamerikas, wie eine unverdächtige Quelle bestätigt: das World Factbook des US-Geheimdienstes CIA“ (S. Schöpp in der Süddeutschen Zeitung 2006). Unerwähnt bleibt hier, dass die CIA wie oben ausgeführt ihr World Factbook zum großen Teil mit Daten der UN bestreitet, die diese wiederum oft ungeprüft von den 
nationalen Statistikämtern übernimmt: „These data are presented as reported by national statistical authorities to the Demographic Yearbook of the Statistics Division of the United Nations Department of Economic and Social Affairs“ (United Nations 2020).

Viele deutsche Medien übernehmen die gefälschten kubanischen Statistiken ungeprüft. „Heute wird in Kuba jeder satt, gibt es keine Obdachlosen mehr, bietet der Staat Bildung kostenlos für jedermann. Er hat das beste Gesundheitssystem in ganz Lateinamerika aufgebaut" (Die Zeit 1987). Dieses sozialistische Wunder schlägt sich nieder ,zum einen in der kostenlosen medizinischen Betreuung der kubanischen Bevölkerung. Um deren Wohl kümmern sich derzeit mehr als 62.000 Mediziner. Die Ärztedichte ist damit fast doppelt so hoch wie in Deutschland. Die Erfolge sind meßbar: Die Säuglingssterblichkeit in Kuba, sie lag vor der Revolution bei 66 Todesfällen je 1000 Neugeborenen, sank auf eine Rate von 9, die niedrigste aller Länder Lateinamerikas“ (Der Spiegel 1999). Oder wie die Süddeutsche Zeitung ein Jahr später den SPD-Staatssekretär Wolf-Michael Catenhusen vom Bundesforschungsministerium nach einem Besuch des Centro de Ingeniería Genética y Biotecnología in Havanna zitiert: „Kuba zeigt auf diesem Gebiet Forschungsleistungen, die das Niveau der entwickelten Industrieländer aufweisen“" (Allhoff 2000).

Hier fühlt man sich an die Industrieroboter der DDR erinnert.

„Wie erklärt sich das Medizin-Wunder der Antillenrepublik? An erster Stelle durch ein Gesundheitssystem, das auch nach deutschen Maßstäben sehr gut abschneidet. 62.000 Mediziner zählt das Elf-Millionen-Volk - die Ärztedichte ist damit nahezu doppelt so hoch wie in Deutschland. Die durchschnittliche Lebenserwartung der kubanischen Bevölkerung liegt heute bei 76 Jahren und ist damit so hoch wie in den Staaten der Ersten Welt“" (Allhoff in Süddeutsche Zeitung 2000). „Was beispielsweise die Säuglingssterblichkeit betrifft, bewegt sich Kuba auf europäischem Niveau - keine Selbstverständlichkeit in der Region." (Schoepp in Süddeutsche Zeitung 2006). „Trotz aller Armut ist die Lebenserwartung heute mit fast 80 Jahren höher als in den USA, die Säuglingssterblichkeit geringer. Praktisch alle Kinder beenden das 9. Schuljahr. Nirgendwo in Lateinamerika gibt es so wenige Morde und Gewaltverbrechen“ (Die Zeit 2011). „Das Gesundheitssystem des Karibikstaats gehört zu den besten der Welt" (Stern 2013).

Warum dann ließ sich Castro, als er ernsthaft erkrankte, Ärzte aus Madrid einfliegen? Ein „Chefchirurg des Gregorio-Marañón-Krankenhaus in der spanischen Hauptstadt ... war auf Bitten der kubanischen Führung nach Havanna gereist, um Castro zu untersuchen. Dessen Zustand beschrieb der Mediziner als ,stabil“", liest man in dem österreichischen Wochenmagazin NEWS (2006). „Der Mediziner war mit einer von der kubanischen Regierung gecharterten Maschine nach Havanna geflogen, um Castro zu untersuchen. Er habe hochmodernes medizinisches Material mitgenommen, das auf der Karibikinsel nicht verfügbar sei, teilte die Madrider Gesundheitsbehörde mit. Bereits seit Juni werde Castro mit Medikamenten aus Spanien versorgt, hieß es weiter".

Auch der Grünen-Politiker Tom Koenigs wünscht sich „,das kubanische Gesundheitssystem ... für alle lateinamerikanischen Staaten, nein: für alle amerikanischen Staaten“ (Interview in der Frankfurter Rundschau, siehe Hebel und Schwarzkopf 2014). Aber hat er schon einmal ein kubanisches Krankenhaus von innen gesehen? 
„Verwandte der Kranken bringen Eimer oder Blechdosen, Seife, frische Handtücher und Bettwäsche in die gewöhnlichen Hospitäler mit, um ein Minimum an Hygiene herstellen zu können. Es gilt, Fäkalien und Blut wegzuschaffen, da die Toiletten und Abflüsse oft verstopft sind, und Patienten wollen ja auch einmal unter die Brause. Von den Wänden eines früheren Herzeigehospitals lösen sich Staubwolken und fallen Kacheln herunter, weil bei der Renovierung zu viel Sand und Zement für die Verschönerung des Altstadtkerns von Havanna abgezweigt worden war" (Widmann 2012, S. 152). Auch in Leciejewski (2020) wird an Hand von Vergleichen verschiedener kubanischer Statistiken die Lüge des „kubanischen Medizinwunders“ nachgewiesen.

Wer ... keine Verwandten in den USA oder in Europa hat und auch sonst keinen Zugang zu fremder Valuta, ist gesundheitspolitisch ein Bürger zweiter Klasse. In einer Klinik für gewöhnliche Kubaner kann der Arzt einem Patienten mit Bronchitis keine Antibiotika verschreiben - die sind in gewöhnlichen Apotheken nicht erhältlich. Sogar Aspirin ist doch nur mühsam aufzutreiben. Und der Unterschied zwischen den ausländischen und den einheimischen Patienten ist der überwiegenden Mehrheit der Kubaner nur allzu geläufig - er ist so auffällig und schmerzhaft wie die Apartheid zwischen Touristen und Einheimischen (Widmann 2012, S. 153).

Kaum Erwähnung in den westlichen Medien finden auch die Gründe, warum so viele Ärzte aus Kuba Dienst in Entwicklungsländern tun. Die häufigste mediale Reaktion darauf ist Zuspruch und Bewunderung. Wie aber in Leciejewski (2020) ausführlich gezeigt, verlässt die Mehrzahl der kubanischen Ärzte keinesfalls freiwillig das Land; sie werden in einer Art modernem Sklavenhandel sozusagen an die Meistbietenden verkauft; ihr Mehrverdienst im Ausland fließt größtenteils an den Staat.

Auf einer höheren Organisationsebene ist etwa die Friedrich-Ebert-Stiftung als Quelle von irreführenden Informationen zu Kuba zu kritisieren. Sie unterhält seit den 1980er-Jahren Kontakte und empfängt oft Delegationen aus Kuba, arbeitet aber selbst von den Nachbarinseln aus. „Vor dem Hintergrund der Reformbemühungen Kubas fokussiert sich die Projektarbeit der FES thematisch auf zentrale Fragen eines nachhaltigen Entwicklungsmodells, die Sozialpolitik, die Wirtschaftspolitik und den Arbeitsmarkt, auf Fragen lokaler Entwicklung sowie auf die internationale Politik,“ ist auf Ihren Netzseiten zu lesen (Friedrich-Ebert-Stiftung 2021). „Die wichtigsten Partner sind die Internationale Abteilung des Zentralkomitees der Kommunistischen Partei, wirtschafts- und außenpolitische Thinktanks sowie verschiedene Institute u. a. der Universität Havanna.“

Unter den mit der Universität Havanna erarbeiteten Dokumenten findet sich ein Diskussionspapier in spanischer Sprache mit dem Titel „Blick auf die Wirtschaft Kubas“ („Miradas a la economía cubana“, Friedrich-Ebert-Stiftung 2017); es steht an Blauäugigkeit und Realitätsverweigerung den entsprechenden Schriften des DIW zur DDR kaum nach. Fünfzehn kubanische Wissenschaftler und eine Wissenschaftlerin aus Uruguay, alle an der Universität von Havanna tätig, legen hier eine Analyse der kubanischen Wirtschaft vor. Weiß denn die Ebert-Stiftung nicht, dass kein Angestellter einer Universität in Kuba, ohne seinen Arbeitsplatz zu gefährden, an einem Papier mitarbeiten darf, das die eigene Wirtschaft mit westlichen Instrumentarien 
analysiert (beispielsweise im Außenhandel die Unterscheidung von Brutto und Netto, Verschuldung, Schuldendienst, Devisenreserven, Deviseneinnahmen u. a.m.) und vielleicht sogar kritisiert? Vergleichsweise unkritisch basieren die Analysen weitgehend auf der offiziellen kubanischen Statistik. Gleiches gilt für die verwendete Literatur. Träfen die dort getätigten Angaben zu den Steigerungsraten der kubanischen Wirtschaft (BIP) zu, wäre Kuba heute eine prosperierende Gesellschaft wie die Bundesrepublik. In keinem sozialistischen Staat wurden jemals die Ziele auch nur eines einzigen Jahresplanes erreicht, die der Fünf-jahrespläne wurden permanent angepasst, also nach unten korrigiert, ohne dies offenzulegen.

Längerfristige Ziele, wie ein in dem Ebert-Papier gefeierter kubanischer Plan über 15 Jahre, gab es gelegentlich auch. So sollte die DDR in zwei Siebenjahresplänen die Wirtschaft der Bundesrepublik pro Kopf überholen (ohne sie einzuholen, nach einem bekannten Bonmot von Walter Ulbricht aus den 1960ern). Insgesamt sind von November 2019 bis Dezember 2020 neun auf den Netzseiten der EbertStiftung aufgelistete Broschüren zur Wirtschaft und Gesellschaft Kubas erschienen, die sich in ihren statistischen Teilen immer auf die staatlichen ONEI-Statistiken berufen, im Einzelfall auch auf CEPAL-Statistiken (Die Wirtschaftskommission für Lateinamerika und die Karibik [spanisch: Comisión Económica para América Latina y el Caribe, CEPAL, englisch: Economic Commission for Latin America and the Caribbean, ECLAC] ist eine Organisation der Vereinten Nationen und verantwortlich für die Förderung der wirtschaftlichen und sozialen Entwicklung dieser Region), deren Zahlen jedoch ebenfalls auf ONEI basieren, und die allein schon deshalb wissenschaftlich wertlos sind.

Im Literaturverzeichnis der Beiträge sind kaum Autoren außerhalb Kubas oder gar Kubakritiker vertreten. Dabei gibt es in einigen lateinamerikanischen Staaten und insbesondere in den USA etliche emigrierte kubanische Ökonomen mit regelmäßigen einschlägigen Beiträgen zu Kuba, die aber auf Missachtung stoßen. Dieser Eindruck einer gewissen Blindheit den wahren Problemen des Landes gegenüber verfestigt sich durch die Eigenvorstellung der Kuba-Arbeit der Friedrich-Ebert Stiftung auf deren Netzseiten. Zwar kommt man nicht umhin, auch einige allzu offensichtliche Defizite anzusprechen, aber ansonsten wird das wahre Ausmaß des ökonomischen Desasters eher kaschiert: „Unter Raúl Castro wurde ein Prozess der ,Aktualisierung ‘ des Wirtschafts- und Sozialmodells Kubas eingeleitet, der bis heute andauert. Durch die kontrollierte Einführung marktwirtschaftlicher Elemente soll schließlich mit einem gemischten System aus Markt- und Planwirtschaft eine Art sanfter Übergang ermöglicht werden, der das Land in die Weltwirtschaft zurückführt, ausländische Direktinvestitionen anzieht sowie Export und Wachstum ankurbelt. Kuba hat sich seither stark verändert, jedoch fehlen weitere Anstrengungen, um die notwendigen wirtschaftlichen Grundlagen für mehr gesellschaftlichen Wohlstand zu schaffen. Die Erfolge in für die Entwicklung wichtigen Bereichen - wie bei der universellen Gesundheitsversorgung, im Bildungssystem, bei der sozialen Absicherung und der öffentlichen Sicherheit - kann Kuba durch seine wirtschaftliche Verfassung immer weniger nutzen. Die im Vergleich zu vielen anderen Ländern erreichten hohen Standards erodieren sogar, vormals homogene soziale Strukturen differenzieren sich.“ (Friedrich-Ebert-Stiftung 2021).

Diese hohen Standards wurden nie erreicht, also können Sie auch nicht erodieren. 


\section{Ausblick}

Der hier beschriebene Tendenz zum Missbrauch von Daten stehen nicht nur nachprüfende Statistiker in nichtsozialistischen Ländern, sondern auch neue Techniken des Big Data Managements entgegen. So konnte etwa Thanh-Long Huynh von der in Paris ansässigen Beratungsfirma QuantCube nachweisen, dass der trotz Corona und anders als in westlichen Ländern in der Volksrepublik China im ersten Halbjahr 2020 ausgewiesene weitere Wirtschaftsaufschwung auf statistischer Manipulation beruhen muss: So sind etwa die von der chinesischen Amtsstatistik gemeldeten Zahlen zu Import und Export nicht verträglich mit den Fahrtrouten von rund 80.000 Güterfrachtern weltweit, die QuantCube über Satellit verfolgt und von denen viele auch chinesische Häfen anlaufen (Bidder 2020). Eine andere Firma namens Earthcube verfolgt per Satellit den Rauchausstoß aus Fabrikschornsteinen oder das Verkehrsaufkommen auf Autobahnen und ermittelt daraus Indikatoren für wirtschaftliche Aktivität (Le Figaro 2020).

Als weiteres vergleichsweise unbestechliches Maß der Wirtschaftsaktivität haben andere Beobachter im Fall von China die Einnahmen aus der Mehrwertsteuer herangezogen. Die sind nicht immer mit den von den Provinzbehörden nach Peking gemeldeten Zahlen zum Wirtschaftswachstum kompatibel. Die werden gerne übertrieben, da es für die verantwortlichen Funktionäre der Kommunistischen Partei von Vorteil für ihre Karriere ist, wenn sie die vorgegebenen Wachstums- und Investitionsziele übertreffen (Lyu et al. 2018). Die Einnahmen aus der Mehrwertsteuer dagegen lassen sich schlechter manipulieren und nicht übertreiben, da von den Empfängern auf Heller und Pfennig nachzurechnen.

Auch die ersten chinesischen Zahlen zur Corona-Mortalität hielten der Kritik der internationalen Datenbeobachtergemeinde nicht stand. „Mit einem Federstrich hat die chinesische Regierung die Todeszahlen um die Hälfte erhöht" (Sinn 2020, S. 17). Dieser Federstrich war nötig, weil die von der chinesischen Statistik gemeldeten Mortalitätsziffern mit den übrigen weltweit beobachteten Raten nicht in Einklang zu bringen waren. So erweist sich das Internet im Verein mit der immer leichteren Verfügbarkeit von Massendaten aller Art für die Amtsstatistik als zweischneidiges Schwert. In seriösen Systemen, und das ist wohl in Europa die große Mehrheit, kann sie helfen, traditionelle statistische Erhebungsverfahren zu beschleunigen oder zu korrigieren. Wie man hier die Übernahme der Daten organisiert, ohne die gewohnten Qualitätsmaßstäbe zu gefährden, ist zur Zeit ein heißes Forschungsfeld (siehe Kühnemann (2021) für einen aktuellen Überblick). Für die schwarzen Schafe dagegen, die nach einem bekannten Bonmot die Statistik benutzen wie ein Betrunkener einen Laternenpfahl: zur Stütze ihres Standpunkts und nicht zu Beleuchtung eines Sachverhalts, steigt damit die Gefahr der Entdeckung beträchtlich an.

Begleitet werden müssten diese Kontrollen aber auch von einem besseren Verständnis für statistische Grundprinzipien in der allgemeinen Bevölkerung. Hier gibt es didaktische Ansätze etwa in der Aktion Unstatistik des Monats (siehe Bauer et al. 2014) oder in der Arbeitsgemeinschaft Statistical Literacy der Deutschen Statistischen Gesellschaft (siehe Krämer et al. (2019) und die Aufsätze in dem einschlägigen Sonderheft des Wirtschafts- und Sozialstatistischen Archivs). Dieser Weg ist 
mühsam, denn speziell in Deutschland scheinen viele ansonsten durchaus gebildete Menschen auch noch stolz darauf zu sein, von Mathematik und Zahlen wenig $\mathrm{zu}$ verstehen. Auch wenn viele das Wort Paradigmenwechsel inzwischen vielleicht nicht mehr hören können: Hier wäre er tatsächlich einmal angebracht.

Danksagung Wir danken Ina-Marie Berendes, Maxime Faymonville und Kai-Robin Lange für unermüdliche Hilfe bei der Literaturrecherche, Daniel O'Donnell und Matthias Reister für Aufklärung betreffend die Modalitäten des internationalen Amtsdaten-Transfers, und einem Herausgeber sowie zwei anonymen Gutachtern für zahlreiche Hinweise und Ergänzungen zu einer früheren Fassung dieses Papiers.

Funding Open Access funding enabled and organized by Projekt DEAL.

Open Access Dieser Artikel wird unter der Creative Commons Namensnennung 4.0 International Lizenz veröffentlicht, welche die Nutzung, Vervielfältigung, Bearbeitung, Verbreitung und Wiedergabe in jeglichem Medium und Format erlaubt, sofern Sie den/die ursprünglichen Autor(en) und die Quelle ordnungsgemäß nennen, einen Link zur Creative Commons Lizenz beifügen und angeben, ob Änderungen vorgenommen wurden.

Die in diesem Artikel enthaltenen Bilder und sonstiges Drittmaterial unterliegen ebenfalls der genannten Creative Commons Lizenz, sofern sich aus der Abbildungslegende nichts anderes ergibt. Sofern das betreffende Material nicht unter der genannten Creative Commons Lizenz steht und die betreffende Handlung nicht nach gesetzlichen Vorschriften erlaubt ist, ist für die oben aufgeführten Weiterverwendungen des Materials die Einwilligung des jeweiligen Rechteinhabers einzuholen.

Weitere Details zur Lizenz entnehmen Sie bitte der Lizenzinformation auf http://creativecommons.org/ licenses/by/4.0/deed.de.

\section{Literatur}

Aleshina N, Redmond G (2005) How High is Infant Mortality in Central and Eastern Europe and the Commonwealth of Independent States? Popul Stud 59:339-354

Allhoff M (2000) Biotechnologie unter Palmen. Auf der Zuckerrohrinsel Kuba arbeiten Genforscher weltweit an vorderster Front. In: Süddeutsche Zeitung (Ausgabe Deutschland. 5. September), S V2/9

Bauer Th, Gigerenzer G, Krämer W (2014) Warum dick nicht doof macht und Genmais nicht tötet. Über Risiken und Nebenwirkungen der Unstatistik. Campus, Frankfurt am Main

Bidder B (2020) Frisierte Wirtschaftsdaten. Weltwirtschaftswundern über China. www.spiegel.de/ wirtschaft/china-und-sein-corona-comback-zweifel-an-den-wirtschaftsdaten-a-3832b91c-3fe74087-af78-3c1ac94eb58a.. Zugegriffen: 27. Aug. 2020

Bomsdorf E, Krämer W (2011) Lenin und die Volkszählung in Russland 1920. AStA Wirtsch Sozialstat Arch 5(3):163-178

Buck HF (1998) Die DDR-Statistik: Manipulation zur Festigung der Diktatur. Orientierungen Wirtsch Gesellschaftspolit 77:56-61

Central Intelligence Agency (2020) CIA the world the world Factbook. www.cia.gov/library/publications/ the-world-factbook/geos/cu.html. Zugegriffen: 27. Aug. 2020

Computerwoche (1982) DDR mit ehrgeizigen Plänen bis 1985: 45000 Roboter für den Rationalisierungsschub, 12. März. https://www.computerwoche.de/a/ddr-mit-ehrgeizigen-planen-bis-1985-45000-roboter-fuer-den-rationalisierungsschub, 1182133; Zugegriffen: 5. Mai 2021

Cornelsen D (1984) Erfolgreiche Produktionsanstrengungen: die Lage der DDR Wirtschaft zur Jahresmitte 1984. DIW Wochenber 32:391-399

Cornelsen D (1989) DDR-Wirtschaft im ersten Halbjahr 1989. DIW Wochenber 31:310-315

Cornelsen D, Melzer M, Scherzinger A (1984) DDR-Wirtschaftssystem: Reform in kleinen Schritten. Vierteljahreshefte Wirtschaftsforsch 2:200-223

Der Standard (2006) Vatikan hat statistisch höchste Kriminalitätsrate der Welt, 18. Januar

Der Spiegel (1999) Biobrom in der Karibik, Nr. 27, 4. Juli

Deutsches Institut für Wirtschaftsforschung (2009) Nachruf auf Doris Cornelsen. Wochenbericht 901:51-52

Die Welt (2008) Jeder achte Deutsche ist arm, 18. Mai 
Die Welt (2014) Fast jeder sechste Deutsche von Armut bedroht. https://www.welt.de/wirtschaft/ article133727719/Fast-jeder-sechste-Deutsche-von-Armut-bedroht.html. Zugegriffen: 27. Aug. 2020

Die Zeit (1987) Medaille für Maria, 8. Mai

Friedrich-Ebert-Stiftung (2017) Mirandas a la economía cubana. Friedrich-Ebert-Stiftung, Düsseldorf

Friedrich-Ebert-Stiftung (2021) Die Arbeit der FES auf Kuba. www.fes.de/referat-lateinamerika-undkaribik/karibik/kuba. Zugegriffen: 21. Jan. 2021

Giesselmann M, Luekemann L (2017) Zunehmende Armut in Deutschland: Empirisch gesichertes Faktum oder Mythos? Gesellsch Wirtsch Politik 1:23-25

Gourbin C, Masuy-Stroobant G (1994) Are live and stillbirths comparable all over europe? Legal definitions and vital registration data processing. International institute for vital registration and statistics. Technical Paper No. 56

Grotlüschen A, Riekmann W (2011) leo. - Level-One Studie. Literalität von Erwachsenen auf den unteren Kompetenzniveaus. Presseheft. Universität Hamburg, Hamburg. https://leo.blogs.uni-hamburg. de/wp-content/uploads/2011/12/leo-Presseheft_15_12_2011.pdf. Zugegriffen: 27. Aug. 2020

Hacker J (1992) Deutsche Irrtümer. Schönfärber und Helfershelfer der SED-Diktatur im Westen. Ullstein, Berlin

Hebel S, Schwarzkopf A (2014) Der weiche Weg ist möglich. Der Grünen-Politiker und Menschenrechtsexperte Tom Koenigs über Rebellionen und Revolutionen, die Rechtfertigung von Gewalt und das Prinzip der Schutzverantwortung. In: Frankfurter Rundschau (Deutschlandausgabe, 19. Juli): 24-25

Hollander P (1981) Political pilgrims. Routladge, Oxford

Kockel K (1992) Ausgewählte Probleme der früheren DDR-Statistik. Allgemeines Statistisches Arch 76:1-14

Koning P, Van Vuuren D (2006) Hidden unemployment in disability insurance in the Netherlands; an empirical analysis based on employer data. CPB Discussion Paper 69. CPB Netherlands Bureau for Economic Policy Analysis,

Koziolek H, Heuer UJ, Friedrich G, Leißing R, Haberland F, Hummel L, Liehmann P, Fischer H, Gerisch R, Salecker W, Krieg J (1970) Die Grundlagen der sozialistischen Wirtschaftsführung in den Werken Lenins und ihre aktuelle Bedeutung. Beiträge anlässlich des 100. Geburtstages W. I. Lenins. Dietz, Berlin

Krämer W (2000) Armut in der Bundesrepublik. Zur Theorie und Praxis eines überforderten Begriffs. Campus, Frankfurt am Main

Krämer W (2003) Der Nürnberger Trichter. Oder wer zählt die Arbeitslosen. Kursbuch 152:93-102

Krämer W (2013) Kalte Enteignung. Wie die Euro-Rettung uns um Wohlstand und Renten bringt. Campus, Frankfurt am Main

Krämer W, Quatember A, Schüller K (2019) Vorwort zum Sonderheft „Statistical Literacy“ des Wirtschafts- und Sozialstatistischen Archivs. AStA Wirtsch Sozialstat Arch 13((3-4), Sonderheft Statistical Literacy):189-191

Kühnemann H (2021) Anwendungen des Web-Scraping in der amtlichen Statistik. AStA Wirtsch Sozialstat Arch 5:5-25

Le Figaro (2020): Fumées d'usines et recherches internet: l'économie en quête de nouvelles boussoles, 10. Juni

Leciejewski K (2020) Kuba - Der Zusammenbruch: Wie ein Paradies seine Zukunft verspielt. Verlag Königshausen und Neumann, Würzburg

Von der Lippe P (1995) Die gesamtwirtschaftlichen Leistungen der DDR-Wirtschaft in den offiziellen Darstellungen. Die amtliche Statistik der DDR als Instrument der Agitation und Propaganda der SED. In: Deutscher Bundestag (Hrsg) Materialien der Enquête-Kommission „Aufarbeitung von Geschichte und Folgen der SED-Diktatur in Deutschland“, Bd. II/3. Suhrkamp, Frankfurt am Main, S 1973-2193

Von der Lippe P (1996) Die politische Rolle der amtlichen Statistik in der ehemaligen DDR. Jahrb Natl Okon Stat 215:641-662

Lyu C, Wang K, Zhang F, Zhang X (2018) GDP management to meet or Beat growth targets. J Account Econ 66:318-338

Marrero L (1957) Geografía de Cuba. Editorial Selecta Havanna

Mertens L (1998) Die geheime Kriminalstatistik der DDR. Kriminalistik 52:103-106

Mesa-Lago C (2009) Economic and social balance of 50 years of Cuban revolution. Cuba Transition 19:368-382

News (2006) Spanischer Arzt untersucht Kubas Staatschef: Fidel Castro leidet nicht an Krebs, 26. Dezember. https://www.news.at/a/spanischer-arzt-kubas-staats-fidel-castro-krebs-160179. Zugegriffen: 5. Mai 2021 
Pérez-López J, Mesa-Lago C (2009) Cuban GDP statistics under the special period: discontinuities, obfuscation, and puzzles. Cuba Transition 19:153-167

Rauch H (1959) Die Verbesserung des Arbeitsstils der Staatlichen Zentralverwaltung für Statistik. Stat Prax 4:161-165

Roesler J (2019) Zwischen Realitätsakzeptanz und Planillusion. Deutschland-Archiv, 15.09.2019. www. bpb.de/297073. Zugegriffen: 19. Jan. 2021

Schoepp S (2006) Permanenter Ausnahmezustand. Touristen retten Kubas Wirtschaft. In: Süddeutsche Zeitung (Ausgabe Deutschland, 9. August), S 2

Sen A (2011) Armut als Mangel an Verwirklichungschancen. Wege zu Gerechtigkeit und Solidarität in der Marktwirtschaft. dtv, München

Sensch J (2007) Die Kriminalitätsentwicklung in der ehemaligen DDR anhand ausgewählter Daten der amtlichen DDR-Kriminalstatistik. GESIS Datenarchiv, Köln ZA8268 Datenfile Version 1.0.0. https:// doi.org/10.4232/1.8268. Zugegriffen: 14. Aug. 2020

Sinn HW (2020) Der Corona-Schock. Wie die Wirtschaft überlebt. Herder, Freiburg

Statistisches Bundesamt (1993) Wohnungsbau und Wohnungsbestand 1970 bis 1990. Sonderreihe mit Beiträgen für das Gebiet der ehemaligen DDR, Heft 2. DeStatis, Wiesbaden

Stern (2013) Was macht Ärzte zum Exportschlager? Nr. 37, 5. September

Unesco (2020) Global education monitoring report. Unesco, Paris

United Nations (2019) 2018 Demographic Yearbook. United Nations, New York

United Nations (2020) Population and vital statistics report. United Nations, New York

United Nations Statistics Division (2020) SDG Indicators. https://unstats.un.org/sdgs/indicators/database/. Zugegriffen: 27. Aug. 2020

Widmann C (2012) Das letzte Buch über Fidel Castro. Hanser, München

Woods CR, Winders-Davis D, Duncan S, Myers J, O'Shea M (2014) Variation in classification of live birth with newborn period death versus fetal death at the local level may impact reported infant mortality rate. BMC Pediatr 14:108-124

World Health Organization (1993) International statistical classification of diseases and related health problems, 10. Aufl. Bd. 2. WHO, Genf

Hinweis des Verlags Der Verlag bleibt in Hinblick auf geografische Zuordnungen und Gebietsbezeichnungen in veröffentlichten Karten und Institutsadressen neutral. 